\title{
Strong Cohomological Dimension
}

\author{
by \\ Jerzy DYDAK and Akira KOYAMA \\ Presented by Czesław BESSAGA
}

\begin{abstract}
Summary. We characterize strong cohomological dimension of separable metric spaces in terms of extension of mappings. Using this characterization, we discuss the relation between strong cohomological dimension and (ordinal) cohomological dimension and give examples to clarify their gaps. We also show that $\operatorname{Ind}_{G} X=\operatorname{dim}_{G} X$ if $X$ is a separable metric ANR and $G$ is a countable Abelian group. Hence $\operatorname{dim}_{\mathbb{Z}} X=\operatorname{dim} X$ for any separable metric ANR $X$.
\end{abstract}

1. Introduction. Strong cohomological dimension was introduced by Kodama [11] as an inductive dimension in cohomological dimension theory for compacta, and we generalize it to separable metric spaces. All spaces considered in this note are assumed to be separable and metrizable.

Definition 1. $X$ has strong cohomological dimension at most $n$ with respect to an Abelian group $G$, written $\operatorname{Ind}_{G} X \leq n$, provided that for any pair of a closed subset $A$ and an open subset $U$ containing $A$, there exists an open subset $V \subset X$ such that

$$
A \subset V \subset U \text { and } \operatorname{dim}_{G} \partial V \leq n-1 .
$$

We define

$$
\operatorname{Ind}_{G} X=\min \left\{n: \operatorname{Ind}_{G} X \leq n\right\} .
$$

Here $\operatorname{dim}_{G} X$ denotes the cohomological dimension of $X$ with respect to $G$. Namely, $\operatorname{dim}_{G} X \leq n$ means that every map $f: A \rightarrow K(G, n)$ of any closed

2000 Mathematics Subject Classification: 55M10, 54F45.

Key words and phrases: cohomological dimension, strong cohomological dimension, ANR, generalized Eilenberg-Sieklucki theorem.

The first author was supported in part by MEC, MTM2006-0825. The second author was supported in part by Grant-in-Aid for Scientific Research (No. 16540064) from Japan Society for the Promotion of Sciences. 
subset $A$ of $X$ to an Eilenberg-MacLane space admits a continuous extension $F: X \rightarrow K(G, n)$.

Note that in the above definition, if we take a singleton as $A$, we obtain the definition of the small strong cohomological dimension $\operatorname{ind}_{G} X$. It is easily seen that for a separable metric space $X, \operatorname{ind}_{G} X=\operatorname{Ind}_{G} X$. Some of the results in this paper may be generalized to wider classes of spaces, but our interest here is restricted to separable metric spaces.

In [9] we showed that $\operatorname{Ind}_{G} X \leq n$ if and only if for given disjoint closed subsets $A$ and $B$ in $X$, there exists a separator $C$ between $A$ and $B$ such that $\operatorname{dim}_{G} C \leq n-1$. For a nontrivial Abelian group $G$, Kodama [11] provided fundamental properties of strong cohomological dimension and showed the following:

THEOREM 1.1 ([11, Theorem 38-6]). For any nontrivial Abelian group $G$ we have the following:

(1) $\operatorname{dim}_{G} X \leq \operatorname{Ind}_{G} X \leq \operatorname{dim}_{G} X+1$,

(2) $\operatorname{Ind}_{G} X \leq \operatorname{Ind} X=\operatorname{dim} X$,

(3) $\operatorname{Ind}_{G} X \leq 1 \Leftrightarrow \operatorname{Ind} X \leq 1$.

Theorem 1.2 ([11, Lemma 38-9]). For every 2-dimensional compact ANR $X$ and every nontrivial Abelian group $G$, we have the equality $\operatorname{dim}_{G} X$ $=\operatorname{Ind}_{G} X=\operatorname{dim} X=2$.

In connection with the above result, Kodama asked ([11, Problem 38-10]): If $X$ is a compact $A N R$, does the equality $\operatorname{dim}_{G} X=\operatorname{Ind}_{G} X$ hold for every Abelian group $G$ ?

This was answered affirmatively by the authors in [9] using the BorsukSieklucki theorem for cohomological dimension.

BorSUK-SiEKLUCKI THEOREM FOR COHOMOLOGICAL DIMENSION ([3], [9], [1]). Suppose that $X$ is a compact $A N R$ and $G$ is an Abelian group. Let $\left\{X_{\alpha}\right\}_{\alpha \in J}$ be an uncountable family of closed subsets of $X$. If $\operatorname{dim}_{G} X=$ $\operatorname{dim}_{G} X_{\alpha}=n$ for all $\alpha \in J$, then $\operatorname{dim}_{G}\left(X_{\alpha} \cap X_{\beta}\right)=n$ for some $\alpha \neq \beta$.

In this paper we shall give a characterization of $\operatorname{Ind}_{G}$, where $G$ is a countable Abelian group, as an application of the following theorem.

Generalized EilenberG-Borsuk theorem ([4], [6]). Let $K$ be a countable $C W$-complex and let $X$ be a separable metric space. If $K * L$ is an absolute neighborhood extensor of $X$ for some $C W$-complex $L$, then for any map $f: A \rightarrow K$ with $A$ closed in $X$, there exists a continuous extension $f^{\prime}: U \rightarrow K$ of $f$ over an open set $U$ such that $L \in \operatorname{AE}(X \backslash U)$.

Then we shall add several fundamental properties of $\operatorname{Ind}_{G}$ and give several examples to show the gap between strong cohomological dimension and cohomological dimension. 
Another application of the generalized Eilenberg-Borsuk theorem is to prove that $\operatorname{dim}_{G} X=\operatorname{Ind}_{G} X$ for every separable metric ANR $X$ and every countable Abelian group $G$ such that $\operatorname{dim}_{G} X>1$. This is done by methods entirely different from those in [9].

2. Results. We shall give a characterization of $\operatorname{Ind}_{G}$ by using the terminology of extension theory. A space $Y$ is an absolute extensor of a space $X$, denoted by $Y \in \operatorname{AE}(X)$, if every map $f: A \rightarrow Y$ with $A$ closed in $X$ extends over $X$. Thus, $\operatorname{dim}_{G} X \leq n$ means $X \in \operatorname{AE}(K(G, n))$. We denote by $\Sigma X$ the reduced suspension of a pointed space $X$.

For a prime number $p, \mathbb{Z}_{p}$ is the cyclic group of order $p$ and $\mathbb{Z}_{(p)}$ is the group of all rational numbers $m / n$ such that $n$ is relatively prime to $p ; \mathbb{Q}$ is the additive group of rational numbers.

TheOrem 2.1. Suppose $X$ is a separable metric space, $G$ is a countable Abelian group and $n>1$. Then the following conditions are equivalent.

(1) $\operatorname{Ind}_{G} X \leq n$.

(2) $\Sigma(K(G, n-1)) \in \mathrm{AE}(X)$.

Proof. $(2) \Rightarrow(1)$. This follows from the generalized Eilenberg-Borsuk theorem. Namely, given disjoint closed subsets $A$ and $B$ in $X$, the map $A \cup B \rightarrow$ $S^{0}=\{-1,1\}$ extends over a neighborhood of $A \cup B$ so that $K(G, n-1) \in$ $\operatorname{AE}(X \backslash U)$. In particular, $\operatorname{dim}_{G} \partial U \leq n$.

$(1) \Rightarrow(2)$. Let $f: A \rightarrow \Sigma K(G, n)$ be a given map of a closed subset $A$ of $X$. There is an open subset $U$ of $X$ with $A \subset U$ and an extension $\tilde{f}: U \rightarrow \Sigma K(G, n)$ of $f$. Since $\operatorname{Ind}_{G} X \leq n$, there is an open subset $V$ of $X$ such that $A \subset V \subset \mathrm{Cl} V \subset U$ and $\operatorname{dim}_{G} \partial V \leq n-1$. Then $\left.\tilde{f}\right|_{\partial V} \simeq 0$. This yields a map $g: X \backslash V \rightarrow \Sigma K(G, n)$. Therefore we can define the extension $F: X \rightarrow \Sigma K(G, n)$ by $\left.F\right|_{V}=\left.\tilde{f}\right|_{V}$ and $\left.F\right|_{X \backslash V}=g$.

Since $S^{1}$ is $K(\mathbb{Z}, 1)$ and so $\Sigma K(\mathbb{Z}, 1) \cong S^{2}$, we have

Corollary 2.2. For every separable metric space $X, \operatorname{Ind}_{\mathbb{Z}} X \leq 2$ is equivalent to $\operatorname{dim} X \leq 2$.

Corollary 2.3. If an infinite-dimensional separable metric space $X$ has $\operatorname{dim}_{\mathbb{Z}} X=2$, then $\operatorname{Ind}_{\mathbb{Z}} X=3$.

For an infinite-dimensional compactum $X$ with $\operatorname{dim}_{\mathbb{Z}} X=2$, by Edwards' cell-like resolution theorem, there exist a compactum $Z$ with $\operatorname{dim} Z=2$ and a cell-like map $f: Z \rightarrow X$. Thus, $\operatorname{Ind}_{\mathbb{Z}} Z<\operatorname{Ind}_{\mathbb{Z}} X$. This yields

COROLlary 2.4. A cell-like map does not preserve integral strong cohomological dimension.

TheOrem 2.5. For each prime $p$ there is a compactum $X$ such that $\operatorname{dim}_{\mathbb{Z}_{(p)}} X=2$ and $\operatorname{Ind}_{\mathbb{Z}_{(p)}} X=3$. 
Proof. Let $X$ be an infinite-dimensional compactum such that $\operatorname{dim}_{\mathbb{Z}_{(p)}} X$ $=2$ and $\operatorname{dim}_{H} X=1$ for every Bockstein group $H \neq \mathbb{Z}_{(p)}$ (see [12, Corollary 3.2]). If $\operatorname{Ind}_{\mathbb{Z}_{(p)}} X=2$, then $X$ has a basis consisting of open sets $V$ such that $\operatorname{dim}_{H} \partial V \leq 1$ for each Bockstein group $H$. In particular, $\operatorname{dim}_{\mathbb{Z}} \partial V \leq 1$, which implies $\operatorname{dim} \partial V \leq 1$. That means $\operatorname{dim} X \leq 2$, a contradiction.

REMARK 1. In connection with Corollaries 2.2 and 2.3 note the following fact: Let $G$ be an Abelian group. If a separable metric space $X$ has $\operatorname{dim}_{G} X=$ $1<\operatorname{dim} X$, then $\operatorname{Ind}_{G} X=2$. For example, take the Pontryagin surface $\Pi_{p}$. Then for any prime number $q \neq p, \operatorname{dim}_{\mathbb{Z}_{q}} \Pi_{p}<\operatorname{Ind}_{\mathbb{Z}_{q}} \Pi_{p}=2$. On the other hand, [9, Corollary 4.8] (or see Theorem 2.11 below) shows that for any finitedimensional separable metric space $X$ and any countable Abelian group $G$, if $\operatorname{dim}_{G} X \geq 2$, then $\operatorname{dim}_{G} X=\operatorname{Ind}_{G} X$. Hence for any separable metric space $X$ and Abelian group $G$, if $2 \leq \operatorname{dim}_{G} X \neq \operatorname{Ind}_{G} X$ and $G$ is countable, then $X$ has to be infinite-dimensional, and if $2 \leq \operatorname{dim}_{G} X \neq \operatorname{Ind}_{G} X$ and $X$ is finite-dimensional, then $G$ has to be uncountable.

Indeed, we do not know whether for each $n \geq 3$ there exist a compactum $X$ and an Abelian group $G$ such that $n=\operatorname{dim}_{G} X<\operatorname{Ind}_{G} X$. Readers may think that the product of the compactum in Theorem 2.5 and the unit interval should be an example. However, the next theorem shows that this is not the case.

Theorem 2.6. If $X$ is a separable metric space and $G$ is an Abelian group, then $\operatorname{Ind}_{G}(X \times \mathbb{I})=\operatorname{dim}_{G}(X \times \mathbb{I})$.

Proof. It is shown in [5, Proposition 1.17] that for any neighborhood $U$ of a closed subset $A$ in $X \times \mathbb{I}$ there is a closed neighborhood $V \subset U$ of $A$ such that $\partial V$ is the union of a locally finite family $\left\{B_{s}\right\}_{s \in S}$ of closed sets, each homeomorphic to a closed subset of $X$. That proves $\operatorname{Ind}_{G}(X \times \mathbb{I}) \leq$ $\operatorname{dim}_{G} X+1$. Since $\operatorname{dim}_{G}(X \times \mathbb{I})=\operatorname{dim}_{G} X+1$, we are done.

Corollary 2.7. Let $X$ be a separable metric space and let $G$ be an Abelian group. If $\operatorname{dim}_{G} X<\operatorname{Ind}_{G} X$, then $\operatorname{Ind}_{G}(X \times \mathbb{I})=\operatorname{Ind}_{G} X$.

REMARK 2. As seen in Remark 1, for distinct prime numbers $p \neq q$, $\operatorname{Ind}_{\mathbb{Z}_{q}} \Pi_{p}=2$. Then, by Dranishnikov's acyclic resolution theorem, there exist a compactum $Z$ with $\operatorname{dim} Z=1$ and a $\mathbb{Z}_{q^{-}}$acyclic map $f: Z \rightarrow \Pi_{p}$. Hence $\mathbb{Z}_{q}$-acyclic maps do not preserve $\operatorname{Ind}_{\mathbb{Z}_{q}}$.

TheOREM 2.8. If a separable metric space $X$ has $\operatorname{dim}_{\mathbb{Q}} X=n>1$, then Ind $_{\mathbb{Q}} X=n$.

Proof. In general, for $m \geq 2$, by [8, Lemma 9.4], $K(\mathbb{Q}, m)$ has the same extension type as $M(\mathbb{Q}, m)$, where for an Abelian group $G, M(G, m)$ is a Moore space of type $(G, m)$. Namely, for a metrizable space $Y, K(\mathbb{Q}, m) \in$ $\operatorname{AE}(Y)$ if and only if $M(\mathbb{Q}, m) \in \operatorname{AE}(Y)$. Now, by the assumption that 
$\operatorname{dim}_{\mathbb{Q}} X=n, M(\mathbb{Q}, n) \in \operatorname{AE}(X)$. Here we may suppose that $M(\mathbb{Q}, n)=$ $\Sigma M(\mathbb{Q}, n-1)$ and $M(\mathbb{Q}, n-1)$ is a countable CW-complex. Therefore for given disjoint closed subsets $A$ and $B$ in $X$, in the same way as in the proof of Theorem $2.1(2) \Rightarrow(1)$, we can find a separator $C$ between $A$ and $B$ such that $M(\mathbb{Q}, n-1) \in \operatorname{AE}(C)$. Then $K(\mathbb{Q}, n-1) \in \operatorname{AE}(C)$. Thus, $\operatorname{Ind}_{\mathbb{Q}} X \leq n$.

REMARK 3. In view of our examples and Theorem 2.8 we may pose the problem of characterizing the class of Abelian groups $G$ such that $\operatorname{Ind}_{G} X=$ $\operatorname{dim}_{G} X$ for every (finite-dimensional) separable metric space $X$. In particular, we may restrict our interest to finite cyclic groups $\mathbb{Z}_{p}$ or finite groups.

Next we give another application of the generalized Eilenberg-Borsuk theorem. Here we consider separable metric ANRs instead of finite-dimensional separable metric spaces. We need the following result from [7].

TheOREM 2.9. Suppose that $X$ is metrizable and $K$ is a connected $C W$ complex such that $\operatorname{dim}_{\pi_{m}(K)} X \leq m$ for each $m \geq 1$. Then $K \in \operatorname{AE}(X)$ in the following cases:

(1) $\pi_{m}(K)=0$ for $m$ sufficiently large,

(2) $\operatorname{dim} X<\infty$,

(3) $X$ is an $A N R$.

Moreover, if $K$ is simply connected, the condition " $\operatorname{dim}_{\pi_{m}(K)} X \leq m$ for each $m \geq 1$ " can be replaced by " $\operatorname{dim}_{H_{m}(K)} X \leq m$ for each $m \geq 1$ ".

Corollary 2.10. For a separable metric ANR $X$, an Abelian group $G$ and $n>1, M(G, n) \in \mathrm{AE}(X)$ is equivalent to $K(G, n) \in \mathrm{AE}(X)$.

Theorem 2.11. Let $G$ be a countable Abelian group. Suppose $X$ is a separable metric ANR or a finite-dimensional separable metric space. If $\operatorname{dim}_{G} X$ $>1$, then $\operatorname{Ind}_{G} X=\operatorname{dim}_{G} X$.

Proof. Suppose $\operatorname{dim}_{G} X=n>1$. As in the proof of Theorem 2.8, it suffices to show that $M(G, n) \in \operatorname{AE}(X)$. In both cases, by Theorem 2.9, the required condition is equivalent to $\operatorname{dim}_{\pi_{m}(M(G, n))} X \leq m$ for each $m \geq 1$, and the second condition is equivalent to $\operatorname{dim}_{H_{m}(M(G, n))} X \leq m$ for each $m \geq 1$ since $n \geq 2$. However, this is clear because $\operatorname{dim}_{G} X \leq n$.

Corollary 2.12. For every separable metric ANR $X$,

$$
\operatorname{dim} X=\operatorname{dim}_{\mathbb{Z}} X=\operatorname{Ind}_{\mathbb{Z}} X .
$$

Those results are generalizations of ones in [13] to separable metric ANRs. However, our proof is completely different.

REMARK 4. Kodama posed another problem relating to $\operatorname{Ind}_{G}$ ([11, Problem 38-8]): Let $f: X \rightarrow Y$ be a closed surjective map between separable metric spaces and let $G$ be an Abelian group. Does the inequality $\operatorname{dim}_{G} X \leq \operatorname{Ind}_{G} Y+\operatorname{dim}_{G} f$ hold? Here $\operatorname{dim}_{G} f=\sup \left\{\operatorname{dim}_{G} f^{-1}(y) \mid y \in Y\right\}$. 
To show that the answer is negative, consider finite-dimensional compacta $Y$ and $F$ such that $\operatorname{dim}_{\mathbb{Z}_{p} \infty}(Y \times F)>\operatorname{dim}_{\mathbb{Z}_{p} \infty} Y+\operatorname{dim}_{\mathbb{Z}_{p} \infty} F$ and $\operatorname{dim}_{\mathbb{Z}_{p} \infty} Y=$ $\operatorname{dim}_{\mathbb{Z}_{p} \infty} F=2\left(\mathbb{Z}_{p^{\infty}}\right.$ is the $p$-torsion of $\left.\mathbb{Q} / \mathbb{Z}\right)$. Let $X=Y \times F$ and let $f: X \rightarrow Y$ be the projection. Now

$$
\operatorname{dim}_{\mathbb{Z}_{p} \infty} X>\operatorname{dim}_{\mathbb{Z}_{p} \infty} Y+\operatorname{dim}_{\mathbb{Z}_{p} \infty} f
$$

and it suffices to invoke Theorem 2.11.

REMARK 5. We do not know whether countability of $G$ is essential in our results. In particular, we can ask whether $\operatorname{dim}_{G} X=\operatorname{Ind}_{G} X$ for every separable metric ANR $X$ and every Abelian group $G$.

3. Remarks. Recently Chigogidze [2] reformulated extraordinary dimension theories as follows.

Definition 2. Let $L$ be a CW-complex and $X$ be a space. We say that

(i) $\operatorname{Ind}_{L} X \leq 0$ if and only if $L \in \operatorname{AE}(X)$;

(ii) $\operatorname{Ind}_{L} X \leq n, n \in \mathbb{N}$, if and only if for every closed subset $A$ and every open neighborhood $V$ of $A$ in $X$, there exists an open subset $U$ such that $A \subset U \subset V$ and $\operatorname{Ind}_{L} \partial U \leq n-1$.

We define

$$
\operatorname{Ind}_{L} X= \begin{cases}\inf \left\{n \geq 0: \operatorname{Ind}_{L} X \leq n\right\} & \text { if such an } n \text { exists, } \\ \infty & \text { if } \operatorname{Ind}_{L} X \not \leq n \text { for any } n \in \mathbb{N} \cup\{0\} .\end{cases}
$$

If we take a singleton for $A$ in the above, we obtain the definition of the small inductive dimension $\operatorname{ind}_{L} X$. Moreover, we set

$$
\operatorname{dim}_{L} X=\min \left\{n \in \mathbb{N} \cup\{0\}: \Sigma^{n} L \in \mathrm{AE}(X)\right\},
$$

where $\Sigma^{n} L$ denotes the iterated suspension of $L$ and $\Sigma^{0} L=L$.

By the definition we easily see that $\operatorname{Ind}_{S^{0}} X=\operatorname{Ind} X$, $\operatorname{ind}_{S^{0}} X=\operatorname{ind} X$ and $\operatorname{dim}_{S^{0}} X=\operatorname{dim} X$ for any space $X . \operatorname{Ind}_{S^{n}} X \leq 0$ if and only if $\operatorname{dim} X$ $\leq n$, and $\operatorname{Ind}_{K(G, n)} X \leq 0$ if and only if $\operatorname{dim}_{G} X \leq n$. Hence $\operatorname{Ind}_{G} X \leq n$ if and only if $\operatorname{Ind}_{K(G, n-1)} X \leq 1$. Therefore we may say that we have considered the problem under what conditions on $X$ and $G$ the inequality $\operatorname{Ind}_{K(G, n-1)} X \leq \operatorname{Ind}_{K(G, n)} X+1$ holds. More generally, we can ask whether Ind $\Omega L X \leq \operatorname{Ind}_{L} X+1$, where $\Omega L$ is the loop space of a pointed CW-complex $L$. Theorem 2.1 essentially says the following:

Theorem 3.1. Let $L$ be a countable $C W$-complex. Then $\operatorname{Ind}_{L} X=$ $\operatorname{Ind}_{\Sigma L} X+1$.

Corollary 2.3 and Theorem 2.5 yield

THEOREM 3.2. There exists a simply connected countable $C W$-complex $L$ and a compactum $X$ such that $\operatorname{Ind}_{\Sigma \Omega L} X \neq \operatorname{Ind}_{L} X$. 


\section{References}

[1] M. Boege, J. Dydak, R. Jiménez, A. Koyama and E. V. Shchepin, Borsuk-Sieklucki theorem in cohomological dimension theory, Fund. Math. 171 (2002), 213-222.

[2] A. Chigogidze, Extraordinary dimension theories generated by complexes, Topology Appl. 138 (2004), 1-20.

[3] J. S. Choi and G. Kozlowski, A generalization of Sieklucki's theorem, Topology Proc. 23 (1998), 135-142.

[4] A. N. Dranishnikov, The Eilenberg-Borsuk theorem for maps into arbitrary complexes, Mat. Sb. 185 (1994), 81-90 (in Russian).

[5] A. N. Dranishnikov and J. Dydak, Extension dimension and extension types, Trudy Mat. Inst. Steklov. 212 (1996), 61-94 (in Russian).

[6] -, - Extension theory of separable metric spaces with applications to dimension theory, Trans. Amer. Math. Soc. 353 (2000), 133-156.

[7] J. Dydak, Cohomological dimension of metrizable spaces I, ibid. 337 (1993), 219-234.

[8] —, Extension theory of infinite symmetric products, Fund. Math. 186 (2005), 39-54.

[9] J. Dydak and A. Koyama, Cohomological dimension of locally connected compacta, Topology Appl. 113 (2001), 39-55.

[10] J. Dydak and J. Walsh, Infinite dimensional compacta having cohomological dimension two: An application of the Sullivan conjecture, Topology 32 (1993), 93-104.

[11] Y. Kodama, Cohomological dimension theory, Appendix in: K. Nagami, Dimension Theory, Academic Press, New York, 1970.

[12] A. Koyama and K. Yokoi, On Dranishnikov's cell-like resolution, Topology Appl. 113 (2001), 87-106.

[13] T. Watanabe, A note on cohomological dimension of approximate movable spaces, Proc. Amer. Math. Soc. 123 (1995), 2883-2885.

Jerzy Dydak

Department of Mathematics

University of Tennessee

Knoxville, TN 37996, U.S.A.

E-mail: dydak@math.utk.edu
Akira Koyama

Department of Mathematics

Faculty of Sciences

Shizuoka University

Oya 836, Shizuoka 422-8529, Japan

E-mail: sakoyam@ipc.shizuoka.ac.jp 